\title{
THOMAS S. KUHN Y SU CONCEPTUALIZACIÓN DEL DESARROLLO DE LA CIENCIA: REFLEXIONES EPISTÉMICAS PARA LAS CIENCIAS SOCIALES
}

\author{
Maricarmen Rivera-Saldaña' \\ "No existe ningún conjunto de categorías neutral, \\ independiente de la cultura, dentro del cual la \\ población-sea de objetos o de acciones-pueda ser \\ descrita; $y$ en este sentido las ciencias naturales \\ no tienen ninguna ventaja sobre las humanas"
}

(Kuhn, 2000a; p.262).

\section{Resumen}

Este escrito indaga en el esquema de análisis que el físico teórico, Thomas S. Kuhn expuso en su obra La estructura de las revoluciones científicas. Se consideran algunas de sus reconceptualizaciones elaboradas durante las siguientes tres décadas de la publicación original, respondiendo a las críticas recibidas y refinando sus posturas. En primera instancia, se revisa el contexto disciplinar de su formación y de sus inquietudes investigativas que le llevan a gestar las primeras ideas que conforman su obra. Se consideran valoraciones sobre la pertinencia de su trabajo en los diversos ámbitos del conocimiento. Luego, se abordan sus tesis centrales, los conceptos y núcleos temáticos desde los cuales argumenta el desarrollo discontinuo y revolucionario del conocimiento científico. También se esboza su propuesta de trabajo para una revisión conceptual de la ciencia desde una perspectiva histórica.

${ }^{1}$ Docente en el Departamento de Psicología de la Universidad de Puerto Rico, Recinto de Río Piedras y en el Departamento de Ciencias Sociales de la Universidad Metropolitana. 
En la parte final, se reconoce la relevancia interdisciplinar de su obra en las discusiones epistemológicas contemporáneas. Se revisan sus argumentos y posturas conceptuales sobre las ciencias sociales y sus particularidades vis a vis las ciencias naturales. Se esbozan algunas coordenadas conceptuales de la discusión de su obra que son sugerentes para repensar la legitimación del quehacer científico en las comunidades científicas de las ciencias sociales que tienen como propósito de estudio comprender los fenómenos humanos en su complejidad. [Descriptores: paradigma, matriz disciplinar, ciencia normal, crisis, revolución científica].

\section{Abstract}

This article examines the analysis framework exposed in the book The structures of the scientific revolutions by the physical theorist Thomas S. Kuhn. I consider some of his reconceptualizations elaborated in the next three decades from the original publication of his work, responding to critics and refining his postures. The first part exposes a review of his research concerns in his disciplinary formation context that shapes the first ideas that form his book. I study some valued reflections of his work pertinence in different fields of knowledge. I address his main thesis, concepts and thematic centers from which he argue the discontinuous and revolutionary development of the scientific knowledge. I also outline his work proposal for a conceptual science review from a historical perspective.

In the final part, I acknowledge the interdisciplinary relevance of his work in the contemporary epistemological discussions. His arguments and conceptual postures over the social sciences and their particularities are considered vis a vis the natural sciences. I expose some conceptual grid references of the debates over his work that is pertinent to rethink the scientific work legitimatization in the social science communities that assume as their purpose comprehend the human phenomena in his complexity. [Keywords: paradigm, disciplinary matrix, normal science, crisis, scientific revolution].

\section{Introducción}

La noción kuhniana de paradigma constituye un referente ineludible en las discusiones contemporáneas sobre la producción del conocimiento científico, y por tanto en las diversas ramas de las ciencias sociales. Esta noción ha sido 
objeto de debates por el sentido inicial en que Kuhn la expuso en su obra principal, La estructura de las revoluciones científicas (en adelante ERC), y por las revisiones consecutivas que hizo del término durante las siguientes tres décadas a su publicación en el 1962. El epígrafe, 27 años después, plasma su reflexión sobre la condición de posibilidad común entre el quehacer científico en las ciencias naturales y en las ciencias sociales: la imposibilidad de producir categorías neutrales para describir los fenómenos bajo estudio (objetos o acciones), al margen del ámbito de la cultura. Con esta postura zanjó el antiguo debate en torno a la primacía de las ciencias naturales versus las ciencias sociales, conocido también como ciencias duras versus ciencias blandas. La aplicabilidad o no aplicabilidad del esquema de análisis kuhniano para las ciencias sociales ha sido fuente de debates desde la década de 1960: el alcance o no de la madurez de las disciplinas, el acuerdo o desacuerdo de un paradigma o la coexistencia de paradigmas. Indudablemente, por más de medio siglo la discusión paradigmática ha mantenido su notoriedad en el terreno epistemológico en los ámbitos de conocimiento en las ciencias sociales. Sin embargo, su utilización frecuente con acepciones que desbordan el análisis kuhniano, sin las clarificaciones terminológicas necesarias puede llevar a confusiones con respecto a cómo se está significando el término. Una revisión exhaustiva de esta noción hace que esta reflexión mantenga un interés vigente al atravesar las tensiones y transformaciones históricas de la discusión sobre la legitimación del conocimiento científico en las ciencias sociales.

El presente trabajo tiene como objetivo indagar en la obra de Thomas S. Kuhn en su faceta como historiador de la ciencia ${ }^{2} y$ su propuesta en torno al desarrollo del conocimiento científico. El análisis tendrá tres niveles de discusión: se explorará el entorno disciplinar en el que se configuró su obra. En el segundo nivel, se abordará la conceptualización de la noción

${ }^{2}$ Kuhn nació en Cincinatti el 18 de julio de 1922 y murió a sus 73 años, el 17 de junio de 1996 (Cambridge, Massachussets). Estudió y se doctoró en Harvard de física teórica. Se desencantó de la física y se interesó por estudiar historia de la ciencia de modo autodidacta, para hacer filosofía. Obtuvo una beca de la Society Fellows que le permitió dedicarse a leer por tres años, proceso que inició su obra ERC. Fue profesor de distintas universidades, como: Harvard, California (Berkeley), en el Center for Advanced Study in the Behavioral Sciences en Stanford (Princeton), y en el Institute of Technology (Massachussets). Recibió diversos reconocimientos (Baltas, Gavroglu, \& Hindi, 2000; Pérez-Ransanz, 1999). 
de paradigma de ERC, se considerarán las revisiones que el mismo Kuhn realiza a lo largo de su carrera (34 años), con el fin de clarificar las dificultades que presentaba la obra inicialmente y para responder a las diversas críticas que recibió ${ }^{3}$. Por último, se hará una valoración de la relevancia de la obra kuhniana en los debates contemporáneos en torno a la legitimidad del conocimiento en las ciencias sociales.

Relevancia de la ERC y ubicación de la obra kuhniana en su entorno disciplinar: Fluctuaciones entre la historia de la ciencia y la filosofía de la ciencia

La tesis kuhniana ha trastocado la imagen tradicional de la ciencia. La obra ERC reviste gran relevancia y es muy citada. Y su autor, el de mayor influencia en las reflexiones científicas contemporáneas porque ha sido la tesis más conocida sobre el desarrollo de la ciencia (López Cerezo, 1998; Pérez Ransanz, 1999). Kuhn (2000c), reconoció que la búsqueda de una mayor compresión sobre el quehacer de la <<empresa científica > en ERC aportó a la transformación de la imagen dominante de la ciencia como estática a una imagen dinámica de la ciencia, incluso fuera del espacio académico. Además, reconoció que cuando la escribió pensó que sus interlocutores serían los filósofos y que se equivocó, inicialmente no fue muy leída por éstos, ni por los científicos. Para su sorpresa tuvo mayor atención e interés en las ciencias sociales, en donde tuvo una muy buena acogida (Baltas, Gavroglu, \& Hindi, 2000; Kuhn, 2000c).

Según Beltrán (1996), su obra ha sido la más citada en la filosofía de la ciencia, aunque en los debates contemporáneos esto no se reconozca unánimemente. La historia, filosofía y sociología de la ciencia son disciplinas que se dividen en un antes y un después de Kuhn. Algunos datos que demuestran esta gran influencia son que, desde su primera publicación, se han vendido más de $1,000,000$ de ejemplares, se ha traducido a 19 idiomas, ha generado una cantidad exorbitante de lecturas secundarias, y la terminología central del análisis ha traspasado al lenguaje corriente (López Cerezo, 1998; Pérez Ransanz, 1999).

A Kuhn se le reconoce como el pensador más sobresaliente de la mitad del siglo veinte, por sus profundas

${ }^{3}$ Este trabajo se basa en la revisión de textos originales de Kuhn entre las décadas de 1960 al 1990. 
intuiciones, los nuevos puntos de partida, y los caminos que se abren con su obra en distintas disciplinas tales como: la psicología, la sociología, la historiografía, la epistemología, la filosofía, la economía del cambio técnico, la teoría literaria, el Trabajo Social, la filosofía de la ciencia, historia de la ciencia, sociología de la ciencia, filosofía de la educación, y en diversos ámbitos de la cultura, entre otras (López Cerezo, 1998; Pérez Ransaz, 1991; Solís Santos, 1998). Por esta razón, ubicar la obra kuhniana en un ámbito disciplinar es una tarea difícil y controversial, debido a que sus elaboraciones han tenido implicaciones en todos los ámbitos donde lo científico ha sido y es estudiado. El debate generado fue amplio y consecutivo, y aun después de la muerte del autor sigue vigente (López Cerezo, 1998; Pérez Ransanz, 1999).

La obra kuhniana se encuentra en una paradoja porque ha sido una gran influencia, pero también ha carecido de una escuela de pensamiento propio, como ha sucedido con autores, como: Karl Popper y Robert Merton. La ausencia de una escuela de pensamiento se podría explicar si se considera que Kuhn no deseaba liderazgo y por la plasticidad de su pensamiento. Se puede hablar de movimiento o de corrientes kuhnianas o postkuhnianas, pero no de una escuela de pensamiento en la disciplina donde se ha abordado el tema, y esto ha dificultado la comprensión del legado de Kuhn en los debates contemporáneos. La $E R C$ ha representado el punto de partida en muchos análisis, pero no ha tenido un estándar fijo debido a que sus recursos conceptuales se han caracterizado por ser flexibles, aunque precisamente esta plasticidad de sus nociones o conceptualizaciones ha generado esa gran influencia en distintas disciplinas (López Cerezo, 1998). Sin embargo, Pérez Ransanz (1999), destaca que a pesar de las críticas que se le pueden hacer a la obra kuhniana, la posibilidad de estudiarla cuidadosamente permite valorar sus "intuiciones básicas," los problemas expuestos y las líneas investigativas que se pusieron de relieve en contraposición de las concepciones tradicionales de las ciencias.

El interés original de Kuhn, luego de completar su doctorado en física teórica, era abordar la ciencia y su enseñanza. Su plan inicial era estudiar historia de la ciencia para realizar filosofía, y de este modo, situarse profesionalmente como historiador. Durante sus años de estudiante siempre 
se caracterizó por tener intereses en diversas temáticas, particularmente en la filosofía, a pesar de que no había podido dedicarse a esto como debió haberlo hecho. Su interés por la filosofía giraba en torno a su inquietud sobre la verdad, es decir, su interés era conocer qué significaba ser verdadero, no era sobre qué era verdadero (Baltas, Gavroglu, \& Hindi, 2000). Kuhn distingue la noción de verdad del entendido de correspondencia de esta con la realidad, es decir, cuestiona la noción de realidad que plantea la filosofía de la ciencia tradicional o dominante porque se refiere a un mundo externo independiente de la mente y de la cultura (Kuhn, 2000c). Sobre la relación entre la historia de la ciencia y la filosofía de la ciencia, expresa que no existe la posibilidad de una formación académica que las una en un solo campo o disciplina de estudio. A pesar de haber sido director de un programa de historia y filosofía de la ciencia, destacó que son campos distintos en sus objetivos, métodos y apreciaciones en torno a las respectivas responsabilidades.

Su propuesta era que, tal como él lo había realizado, se podían ejercer ambas disciplinas, pero sin fundirlas. Entre ambas disciplinas debía de haber un intercambio fructífero, y aunque subrayaba esta posibilidad de interacción entre ambas disciplinas, puntualizaba que esto presentaba dificultades porque los historiadores habian dejado de abordar aspectos técnicos, mientras que los filósofos atendían las cuestiones específicas para determinar qué era lo correcto y lo incorrecto. En este sentido, establece un punto de encuentro entre los filósofos y los científicos, la preocupación continua por lo correcto y lo que no lo es, mientras que los historiadores se aproximan a atender por qué un ser humano piensa cómo lo hace. Señala que revisar un texto con la sola preocupación de lo que tiene correcto o incorrecto pierde de perspectiva otros aspectos que podrían ser relevantes (Baltas, Gavroglu, \& Hindi, 2000). Los cuestionamientos centrales del historiador son: ¿por qué las personas eligen cambiar las creencias que tienen? $Y$ ¿por qué tiene lugar el cambio creciente? El historiador no debe cuestionarse las creencias de las personas en sí mismas, su función es mostrar cómo y por qué se producen los cambios en las creencias del momento y el lugar que se estudia (Kuhn, 2000b; Kuhn, 2000c). Para Kuhn, el objetivo último de la labor de la investigación histórica es hacer narraciones que se inicien desde la ubicación de la escena o puesta en escena, cuidándose 
de utilizar un lenguaje contemporáneo para referirse a ese otro momento histórico que estudia. Dicho de otro modo, no se debe utilizar una terminología científica moderna para referirse a las creencias de la Antigüedad (Kuhn, 2000c).

Su acercamiento a la historia de la ciencia inicia cuando comenzó a trabajar como asistente de James B. Conant, debido a que este le asignó la tarea de presentar un caso de la historia para el curso. En ese momento fue cuando trabajó por primera vez a Aristóteles. Kuhn estudió el tema de la transición de la física aristotélica a la newtoniana, su interés era indagar sobre las creencias anteriores y su racionalidad. Esta tarea fue muy significativa para sus reflexiones posteriores porque a partir de ese momento decidió que quería estudiar historia de la ciencia, e introdujo la noción de cambio revolucionario en su pensamiento uniéndose con la idea que tenía del carácter no acumulativo del desarrollo de la ciencia. En su concepción en torno al quehacer del historiador en la indagación de las creencias de las personas en otras épocas, destacaba la relevancia de explicar elaborando la escena desde un esquema conceptual diferente. En este momento surgió su deseo de escribir ERC (Baltas, Gavroglu, \& Hindi, 2000; Kuhn, 1996d; Kuhn,2000c).

En el proceso de formación intelectual de Kuhn y en el transcurso de la elaboración de $E R C$, hubo autores y autoras de diversos ámbitos disciplinares que fueron relevantes, como: Alexander Koyré, Emile Meyerson, Helene Metzger, Anneliese Mair, Ludwick Fleck, Jean Piaget, la Psicología de la Gestalt, B.L. Whorf, W.V. Quine, Leonard K. Nash, Stanley Cavell, entre otros/ as. Alexander Koyré fue un autor crucial en su formación como historiador de la ciencia, de él reconoció haber aprendido cosas que no había imaginado. A este autor le atribuyó la creación de una nueva imagen de la ciencia plasmada en la $E R C$ (Baltas, Gavroglu, \& Hindi, 2000; Kuhn, 1996b). Conoció a Koyré por su texto Estudios galileanos, y cuando escribió $E R C$ pensó que su postura sobre la historia de la ciencia era internalista. Sin embargo, Koyré, por vía epistolar, le indicó su apreciación de la obra como la unificación entre la historia externa e interna de la ciencia. Kuhn admitió que él mismo no había considerado esa interpretación y que se impresionó de esta valoración por venir de un hombre con gran capacidad en el análisis de las ideas. La apreciación de Koyré resulta interesante porque Kuhn se excusa por haber excluido en su análisis condiciones 
externas del desarrollo de las ciencias que son relevantes, y que debió haber considerado, como: las dimensiones sociales, económicas y sobre el progreso tecnológico. Incluso, sostuvo que su perspectiva en torno al desarrollo de la ciencia fue una aproximación que se quedó en los límites de la vertiente internalista de la ciencia. (Baltas, Gavroglu, \& Hindi, 2000; Kuhn, 1996a; Kuhn, 1996b).

Kuhn reconoció que perteneció a una generación de filósofos/historiadores amateurs cuya formación era científica, pero que sus objetivos filosóficos los llevaron a ocuparse de la historia de la ciencia. Al igual que sus colegas, entendía en ese momento que a través de la historia se podía construir una filosofía de la ciencia distinta de la que planteaba el positivismo lógico o el empirismo lógico u otros empirismos. Su pretensión era que ese nuevo modo de hacer filosofía de la ciencia fuera sobre las observaciones de la vida científica, es decir, estos se percibían "...construyendo una filosofía sobre las observaciones de la conducta científica real"(Kuhn, 2000c;p. 138). Sin embargo, reconoció que dicha perspectiva histórica era el producto de una revisión general de la documentación histórica. El trastocamiento de la imagen de la ciencia estática por la imagen de la ciencia dinámica que se produjo fue producto de la reflexión de los hechos históricos. Según Pérez Ransanz (1999), Kuhn fue el pionero y algunos de los autores involucrados fueron: Norwood Hanson, Paul Feyerabend y Stephen Toulmin. Algunas de las denominaciones para el aclamado movimiento del '60 fueron: Nueva filosofía de la ciencia, Corriente historicista, Teoreticismo, Análisis de cosmovisiones y Filosofía blanda de la ciencia. En la década de1960 se generó un ambiente de cuestionamiento crítico a los supuestos de las posturas clásicas de la filosofía de la ciencia, estos nuevos análisis surgieron desde la historia de la ciencia. No obstante, los filósofos de la ciencia fueron los más sacudidos (López Cerezo, 1998; Pérez Ransanz, 1999).

La concepción de ciencia en la que habían enseñado a Kuhn y a los de su generación fue una que asumía que los hechos eran el punto de partida de la investigación, éstos se obtenían por la observación, eran objetivos e interpretables mediante un proceso humano universal, es decir, para todos por igual. En el proceso de búsqueda, Kuhn y sus colegas se percataron que los hechos no eran independientes de las creencias o de las teorías existentes. La producción de los hechos implicaba el 
conocimiento teórico, además el establecimiento de un diseño en ocasiones requería que se retomaran las concepciones del fenómeno observado. Existían otras dificultades con los procesos interpretativos porque habían diferentes interpretaciones en la aceptación de teorías o leyes, incluso individuos comprometidos con determinada interpretación defendían posicionamientos que violentaban los cánones de ética establecidos y juramentados por la profesión. Kuhn critica la incapacidad de los científicos para reconocer los descubrimientos de sus adversarios, específicamente cuando se evitaba el argumento y se entraba en el ámbito personal dejando los debates de temas científicos como "pelea de gatos" (Kuhn, 2000c; p.134). Sus colegas y él criticaron a la filosofía de la ciencia dominante por centrase en las normas metodológicas atribuyéndoles la capacidad de resistir a las violaciones ocasionales. Kuhn consideró relevantes los aspectos diferenciales entre los individuos (científicos) tales como: los gustos personales o la historia personal, como por ejemplo las recompensas o penalizaciones de financiación o prestigio. Estas fueron las claves de conducta que le guiaron a él y a sus colegas a reformular los acercamientos de la filosofía de la ciencia dominante. Su postura mantuvo la crítica hacia la tradición de la filosofía de la ciencia por asumir un mundo real y el mundo exterior objetivo entre los cuales hay correspondencia, que es el criterio que evaluaba la verdad o probabilidad de verdad. La perspectiva kuhniana entendía que las evaluaciones siempre son indirectas, y a través de esto es que se aplicaban los criterios secundarios tales como: precisión, consistencia con creencias aceptadas, amplitud de aplicabilidad y la simplicidad ubicada en el ojo del observador (Kuhn, 2000c).

Kuhn señaló que aportó en gran medida a este ambiente crítico que se generó en la década de1960. Expuso que la filosofía de la ciencia tradicional ha estado equivocada no sólo con respecto a los métodos, sino también con respecto a la concepción misma de conocimiento, pero esto no debería suponer que en los análisis del desarrollo de la empresa científica se reemplacen la evidencia y la razón por el poder y el interés. Propuso realizar una revisión conceptual en tres dimensiones: que se evalúen los cambios de creencias no la creencia en sí misma, que se reconozca que los cambios a evaluar suelen ser pequeños y que sólo en retrospectiva logran el estatus de piedra angular (proceso circular), y que se debe de 
abandonar la idea de la ciencia como empresa monolítica regida por un solo método. Su propuesta fue una perspectiva histórica o evolutiva que consideró la proliferación de especialidades o subespecialidades como relevantes, considerando aspectos como la cantidad de departamentos de una disciplina y la cantidad de revistas de disciplinas específicas o subgrupos en las disciplinas. Las subespecialidades aumentan las cualidades de precisión, consistencia, amplitud de la aplicación y la simplicidad del conjunto de creencias otorgándole continuidad a las herramientas o técnicas de mayor refinamiento para describir la naturaleza. Lo único posible según el autor es la comparación de conjuntos de creencias que existen en determinada situación histórica (Kuhn, 2000c).

Kuhn reconoció que el manejo de los contenidos abordados en ERC al momento de su publicación era insuficiente y tosco, además de cometer el error de generalizar en exceso. Por tal razón, se dedicó los próximos treinta años a una mayor comprensión y al refinamiento de dichos contenidos, y consideró que solo en los últimos cinco años hizo progresos significativos (Kuhn, 2000b). Expuso su concepción de ciencia en la década del 90 como:

...una estructura compleja pero no sistemática de distintas especialidades o especies, y cada una dedicada a cambiar las creencias vigentes sobre su dominio ... Yo sugiero que puede verse que esta empresa, las ciencias, que entonces deben ser consideradas como una pluralidad, conservan una autoridad muy considerable. (Kuhn, 2000c; p. 147)

Kuhn no asume dicotomía entre la dimensión sociológica de la ciencia y el carácter empírico intrínseco del quehacer científico, ni contrapone la evidencia y la razón versus el poder y el interés. Su perspectiva considera que todos estos aspectos están involucrados en lo que los científicos producen y cómo lo producen. Dicho de otro modo, es necesario repensar la labor científica considerando que el poder y el interés son hechos observados en la praxis científica, y por tanto, son intrínsecos al proceso evolutivo o de desarrollo de la ciencia desde la consideración de la historia personal, gustos, y creencias del científico. 


\section{La conceptualización kuhniana sobre la noción de paradigma}

En ERC el propósito de Kuhn fue denunciar la necesidad de un cambio en el modo de concebir o percibir, y de evaluar los datos de la actividad investigativa científica. Su tesis central afirma que el desarrollo del conocimiento científico se constituye de modo discontinuo, es decir, su desarrollo no es acumulativo. Su propuesta fue puntualizar que la investigación histórica y la investigación sociológica tienen un potencial de desarrollo fecundo para el análisis del desarrollo científico. La revolución historiográfica en el estudio de la ciencia enfatiza en la integridad histórica de su propia época, por la cual, el desarrollo científico no es el producto de la mera acumulación de datos (Kuhn, 1996b).

Desde una mirada general, el análisis de ERC expone distintos momentos de este desarrollo discontinuo que inician con la fase pre-paradigmática a un primer momento paradigmático, en el cual se establece la ciencia normal. La pre-ciencia o etapa preparadigmática es cuando la investigación científica es ambigua porque se yuxtaponen unas posturas versus otras y no está establecido cuáles son las descripciones e interpretaciones de los fenómenos que van a guiar a la comunidad. Esta fase finaliza cuando una de las escuelas en pugna logra el lugar de paradigma estableciéndose la ciencia normal. La ciencia normal es cuando los términos y criterios que rigen la investigación de esa comunidad científica están claramente establecidos de antemano, se establece un consenso sobre el paradigma que regirá la actividad científica. Consecuentemente serán transmitidos estos acuerdos mediante la educación profesional a los sucesores, éstos son: los cuadros conceptuales desde los que se delimitan las zonas y fenómenos para investigar; estos se reducirán a problemas específicos, detallados y profundos; con el fin último de articular las teorías que ofrece el paradigma con los fenómenos estudiados, aumentando el alcance y precisión del paradigma, y consumiéndole casi todo el tiempo a los científicos en esto. El supuesto de la ciencia normal es que ya toda la comunidad conoce cómo es el mundo, es decir, se genera un ambiente de confianza en el cual los científicos obligan a que la naturaleza encaje en los límites del paradigma, esto es lo que se asume como llegar a un nivel de madurez. En la ciencia normal se establece una red de compromisos conceptuales, teóricos, 
instrumentales y metodológicos. La ciencia normal inicia un proceso de cuestionamiento cuando surgen enigmas, éstos son problemas que ponen a prueba el ingenio o habilidad del paradigma para resolverlos. Los enigmas para ser considerados como tales, deben tener más de una solución asegurada, además estos son aislados por la ciencia normal. Cuando los científicos como profesión reconocen los enigmas, estos pasan a ser anomalías. Las anomalías son violaciones a lo que era esperado, es decir, son problemas sin resolver dentro de los parámetros del paradigma vigente ("extravíos de la ciencia normal"). Si la percepción de la anomalía dura mucho tiempo penetrando profundamente, surge una crisis. $Y$ esta surge por la incapacidad de explicación de la ciencia normal, generándose un ambiente de confusión sobre el paradigma y debilitándose la legitimación de las reglas de la investigación normal. La función de las crisis es servir de antesala para la elaboración de nuevas teorías que terminará posteriormente en el cambio fundamental del paradigma. La crisis concatena una revolución científica en la cual surgen distintas posibles teorías alternativas para la solución de los enigmas para ser consideradas como el próximo o segundo momento de establecimiento de un paradigma en la ciencia en cuestión. La revolución científica se caracteriza por los cambios en los compromisos profesionales, quebrando la tradición de la ciencia normal. En el proceso de revolución científica se rechaza una teoría para acoger a otra, cambian los problemas de análisis, cambian las normas, cambian las soluciones, cambia la imaginación científica. La asimilación de una nueva teoría implica la reconstrucción de la teoría anterior y la reevaluación de los hechos anteriores. El nuevo paradigma es un embrión, durante el proceso de transición entre un paradigma y otro se reconstruye el campo, surgen nuevos fundamentos, generalizaciones teóricas, métodos y aplicaciones, por lo cual no es un asunto exclusivo de invención de teorías. El proceso de elección entre paradigmas es un proceso circular (Kuhn, 1996b).

La noción de paradigma es una característica de la época moderna, que se define como un modelo o patrón aceptado que afectará la estructura del grupo practicante, permitiendo una mayor articulación y especificación de la investigación. Su establecimiento en un campo científico representa madurez, por esto la noción de paradigma se relaciona a la de ciencia 
normal. Para que una teoría se acepte como paradigma debe parecer mejor que sus competidoras. Sin embargo, no necesita explicar todos los hechos que se puedan confrontar con ella (en efecto nunca lo hace). El tránsito de una teoría a constituirse en paradigma supone que debe: sugerir los experimentos que se deben realizar; poner punto final a la reiteración constante de fundamentos; generar confianza para animar a los científicos; ocuparse de fenómenos seleccionados de un modo más detallado; diseñar el equipo especial para las tareas usándolo más sistemáticamente; reunir datos y hechos; formular teorias como actividades dirigidas; aumentar la efectividad y la eficiencia. Este proceso de tránsito implica un proceso de conversión de los científicos al nuevo paradigma, por esto las antiguas escuelas desaparecerán eventualmente. Un nuevo paradigma dará una definición nueva y rígida del campo, razón por la cual una vez se establece, no es necesario justificar continuamente los principios del campo ya que las publicaciones se dirigen a los colegas profesionales. Sin embargo, durante la primera aparición de este nuevo paradigma su alcance puede ser muy limitado. Su legitimación se logra porque tiene más éxito que sus competidores en la solución de los problemas agudos de la disciplina. No obstante, la travesía hacia el consenso es un proceso arduo (Kuhn, 1996b).

En respuesta a las críticas, estableció dos clasificaciones del término paradigma: la concepción global o el sentido sociológico del término que, alude al conjunto o conglomerado de creencias, valores, técnicas, etc., que comparten los científicos de determinada comunidad científica. Es decir, son todos aquellos compromisos compartidos del grupo de científicos, denominados como matriz disciplinar. La segunda clasificación del término paradigma se refiere a las soluciones concretas de problemas que, reemplazan en ocasiones las reglas explícitas para resolver los problemas en la ciencia normal, y estos son denominados como ejemplares o "ejemplos estándar de una comunidad" (modelos o ejemplos). Esta acepción del término es la que Kuhn identifica con mayor profundidad filosófica, porque responde a la necesidad de abordar el desarrollo del conocimiento científico cuando este no es regido por reglas explícitas. El proceso de aprendizaje de ejemplares profesionales es un proceso de asimilación en donde el estudiante identifica o reconoce semejanzas en 
la solución de los problemas concretos de la ciencia. Esta clasificación o acepción del término es el que ha propiciado mayores controversias (Baltas, Gavroglu, \& Hindi, 2000; Kuhn, 1996c; Kuhn, 1978). Los ejemplares (paradigmas o conjuntos de paradigmas) son los que guían la praxis científica de los grupos que configuran la comunidad científica. Los ejemplares son la solución de problemas concretos que se asumen como paradigmáticos, estos a su vez forman parte de la matriz disciplinar o compromisos generales (Kuhn, 1996c; Kuhn, 1978).

Kuhn clarifica el propósito inicial del uso del término paradigma en ERC, para abordar e investigar una comunidad científica en la cual, no eran fácilmente identificables las reglas compartidas, y de este modo, explicar la conducta aproblemática del grupo de investigación determinado. Así, llega a establecer que los ejemplos compartidos o ejemplares de una práctica exitosa pueden sustituir la deficiencia de las reglas del grupo (Kuhn, 1996c; Kuhn, 1978). Es entonces cuando los ejemplares pasan a ser los paradigmas, por tal razón esenciales en la investigación de la comunidad científica. Sin embargo, el autor se lamenta de haber utilizado el término paradigma para aludir a los compromisos compartidos del grupo, a lo cual denominó posteriormente como "matriz disciplinar". Kuhn asumió la responsabilidad de las confusiones surgidas, pero sostuvo que los ejemplos compartidos son un referente exitoso en el desempeño de las funciones cognoscitivas cuando existen deficiencias en las reglas de la comunidad científica. Kuhn afirmó que las comunidades científicas tienen reglas compartidas por sus miembros, además expone que otras reglas podrian ser inducidas legítimamente con observaciones del comportamiento de los científicos. Critica a los filósofos de la ciencia por sólo haber atendido los elementos formales de claridad y estructura de las teorías científicas, sin problematizar la relación entre lenguaje y naturaleza. Kuhn critica el fundamento epistémico de los formalistas porque obvian la tarea de analizar el conocimiento científico.

Otra clarificación con respecto a la noción de "paradigma" es que esta fue introducida con una estrecha relación al término de "comunidad científica" (Kuhn, 1996c; Kuhn, 1978). La constitución de un paradigma requiere de la conexión y participación de un grupo de personas de un ámbito científico. El término de paradigma es intrínseco a la existencia de las comunidades científicas en la ciencia 
moderna. Kuhn (2000b), afirma que la ciencia es una actividad comunitaria por definición. El punto de partida kuhniano es una definición intuitiva de comunidad que se remite a quienes practican determinada especialidad científica que tienen como elementos comunes la educación y períodos de aprendizaje, asumiendo la responsabilidad de que se le dé continuidad al conglomerado de metas y objetivos que comparten incluyendo a quienes serán los sucesores (Kuhn, 1978). Las comunidades científicas se caracterizan por producir y validar el conocimiento científico, además por poseer comunicación intragrupal; tener la unanimidad de los juicios profesionales sobre las materias en cuestión; coincidir con la literatura de referencia y como consecuencia tener coincidencias en las lecciones e interpretaciones de esta literatura. La comunicación profesional entre diversos grupos de diferentes materias en una tarea difícil, porque se puede prestar para malas interpretaciones y discrepancias significativas (Kuhn, 1978). La comparación de las actividades de un grupo científico permite establecer las semejanzas y diferencias con otros grupos disciplinarios, incluso con otros campos del mismo grupo (Kuhn, 1996e). La matriz disciplinar es el modo explicativo del carácter aproblemático de los elementos compartidos de la comunicación profesional y de su unanimidad del juicio (Kuhn, 1978).

Kuhn se autocriticó por haber introducido el término de paradigma modo circular cuando se refirió al período preparadigmático y luego en el período postparadigmático del desarrollo de la ciencia (Kuhn, 1996c; Kuhn, 1978). En su texto, Segundos pensamientos sobre paradigmas, se refirió a la noción de paradigma como una conceptualización de relevancia:

Todavía creo que este modelo es típico e importante, pero se lo puede examinar sin hacer referencia a la primera consecución de un paradigma. Sean lo que fueren, los paradigmas son poseídos por cualquier comunidad científica, incluidas las escuelas del llamado "período preparadigmático". Mi fallo en no ver este punto con claridad ha contribuido a que un paradigma semejase ser una entidad o propiedad cuasi-mística que, como un carisma, transforma todo lo infectado por él. Se da una transformación, pero no inducida por la adquisición de un paradigma. (Kuhn 1978; p.13) 
La reelaboración del autor amplia y flexibiliza la noción de paradigma y su esquema categorial para indagar en el desarrollo del conocimiento científico que en sus inicios expuso en ERC. De modo que, su análisis conceptual no debería asumirse como absoluto o cerrado, sino que deberían de considerarse estas reelaboraciones a la luz de la comunidad científica específica.

Adicional a la crítica de las múltiples acepciones del término paradigma de Kuhn, recibió otras críticas: (a) que exageró en la unanimidad de los científicos en un paradigma; (b) que tuvo un exclusivo interés en las grandes revoluciones como las de Copérnico, Newton, Darwin o Einstein; (c) que concibió la crisis como antecesora invariable de las revoluciones; (d) que su alusión a los valores compartidos fue una flaqueza porque glorificó la subjetividad y la irracionalidad, por lo que se le ha acusado de relativista. Sobre la exageración con respecto a la unanimidad de los científicos, expuso que la necesidad de un acuerdo entre científicos dependería de lo que hace la comunidad cuestión. Sobre la crítica con respecto a su concepción de la noción de revolución, en la Posdata destacó que, la misma constituye un cambio especial que implica que los compromisos de la comunidad científica se reconstruyan. Los cambios no tienen que ser grandes o incluso ni tienen que parecerlos para quienes no pertenecen a esta. Cabe destacar, que Kuhn reconoció que la constitución de una comunidad científica se puede constituir con tan solo veinticinco integrantes (Kuhn, 1996c). La relevancia de la concepción revolucionaria del desarrollo científico estriba fundamentalmente en que denuncia la concepción acumulativa de la ciencia, porque el conocimiento que genera no es una mera adición de lo que ya se conoce. En ERC el autor elabora dos tipos de desarrollo científico el normal y el revolucionario (Kuhn, 1996d). El desarrollo normal es el acumulativo que sirve de guía metodológica, por lo cual es aplicable a la mayor parte del que-hacer científico (ciencia normal). El desarrollo revolucionario de la ciencia provee claves únicas para su análisis porque los descubrimientos no son ajustables a los conceptos habituales. El proceso de asimilación de estos descubrimientos trastoca los modos de pensar y describir los fenómenos naturales, constituye un nuevo modo de conectar o integrar las ideas (o los términos) con la naturaleza. Como consecuencia, se establecen nuevas pautas que antes no existían, implicando cambios en las categorías 
taxonómicas. Estas innovaciones o descubrimientos suelen confrontar rechazo o pueden ser ignorados durante largos períodos de tiempo en una ciencia (Kuhn, 1996d; Kuhn, 2000b). La inconmensurabilidad es una disparidad conceptual entre dos especialidades que se han desarrollado paralelamente, esto hace que los profesionales de las especialidades involucradas no se puedan comunicar, resultando difícil la posibilidad de que estas disciplinas puedan producir conocimiento fértil (Kuhn, 2000c). Las nociones más relevantes en ERC son: el <<cambio revolucionario > y la <<inconmensurabilidad >, ambas nociones son el eje o núcleo central de su análisis (Kuhn, 2000b).

La tercera crítica que recibió Kuhn fue en torno a su concepción de crisis como evento ineludible para que se geste una revolución. El autor señaló que su exposición inicial no fue planteada en términos de un criterio absoluto, sino que su elaboración asumió las crisis como preludio habitual. La cuarta crítica, es sobre la categorización de su análisis como subjetivo, irracional y relativista porque hace referencia a los valores compartidos y compromisos profundos entre los hombres de ciencia como parte constitutiva de esta. Los valores compartidos tienen la función de distribuir riesgos garantizando el éxito de la comunidad científica, además estos valores se afectan por los rasgos de personalidad de quienes integran el grupo en cuestión. La labor científica implica riesgos, porque no puede responder a todas las anomalías, pero tampoco puede ignorar todas las anomalías porque de ser así no habría posibilidad de revoluciones (de desarrollo científico). La variabilidad individual tiene una función esencial para la ciencia porque la labor científica requiere riesgos (Kuhn, 1996c). Dicho de otro modo, las revoluciones necesitan de reacciones ante las anomalías, y esto supone un coste personal de los científicos que asumen las anomalías como su quehacer, porque los científicos son formados y recompensados por solucionar los rompecabezas (instrumentales, teóricos, lógicos o matemáticos) de la interfaz del mundo fenoménico y las creencias que tiene la comunidad científica en cuestión. Según Kuhn (2000b), el éxito profesional se mide por el incremento de la precisión del ámbito en el que se apliquen. Sin embargo, los ejemplares o ejemplos profesionales con los cuales los científicos se forman y aprenden a identificar el alcance, la precisión, la simplicidad, etc., cambian al interior 
de la disciplina y también entre estas. Kuhn destacó que la solución de rompecabezas sumerge a los científicos en asuntos políticos y de poder, al interior de las prácticas de solución de problemas concretos, como entre estas prácticas profesionales y la cultura no científica.

La postura que el autor designa como reflexiones de mayor expresión y refinamiento conceptual con respecto al desarrollo del conocimiento científico asume que las etapas evolutivas de un campo o especialidad se construyen sobre la que le precede (aunque no en todos los aspectos). Por lo cual, la etapa predecesora provee los problemas, datos y conceptos que son requisitos necesarios para que emerja la siguiente. Las transformaciones en el vocabulario conceptual son inherentes al proceso de asimilación para que surja la próxima etapa:

... el proceso de transición de la etapa antigua a una nueva se convierte en una parte integral de la ciencia, un proceso que debe ser entendido por el metodólogo interesado en analizar las bases cognitivas para las creencias científicas. Para mí, el cambio de lenguaje es cognitivamente significativo... (Kuhn, 2000b; p. 271)

Las reelaboraciones kuhnianas al igual que estas últimas reflexiones no siempre se han considerado en la amplia gama de la literatura secundaria que ha generado la discusión de la obra inicial. Ha habido una tendencia de polarizar la discusión entre lo objetivo versus lo subjetivo y entre el racionalismo versus el relativismo (Chalmers, 1998; Solís Santos, 1998). Esta acusación de relativista, fue negada por el autor subrayando en innumerables ocasiones que su propuesta de trabajo era precisamente visibilizar aspectos del quehacer científico y de su producción que se obviaban desde la filosofía de la ciencia tradicional. Toda su obra giró en torno a profundizar en la discusión del desarrollo del conocimiento científico considerado la dimensión empírica de la ciencia como parte fundamental de este quehacer.

\section{Valoración de la obra kuhniana: Aportaciones epistémicas para las ciencias sociales}

Según López Cerezo (1998), las aportaciones de Kuhn son fundamentalmente tres: la nueva terminología que introdujo al análisis científico; el carácter histórico y dinámico de la ciencia con situaciones específicas de la ciencia (análisis 
empírico); y su estilo interdisciplinario. Estas aportaciones permiten que su obra se entrecruce con distintas disciplinas como la historia, filosofía y sociología de la ciencia: "... el estilo interdisciplinario se constata en el uso de un marco conceptual enriquecido donde los problemas epistemológicos adquieren una dimensión sociológica y los términos del análisis son compilados de una variedad de disciplinas" (López Cerezo, 1998; p.180). Solís Santos (1998), señala que Kuhn abrió las puertas de la filosofía de la ciencia a la Historia, a la Sociología y a la Psicología. Este autor coincide con López Cerezo (1998), en que la $E R C$ aportó el trastocamiento de distintas disciplinas con respecto a las problemáticas epistémicas, contribuyendo al inconformismo epistémico y ético de la razón. Mientras Solís Santos (1998), reconoce que sus aportaciones sobre la ciencia consistieron en el planteamiento de que el desarrollo científico implica discontinuidades que ocurren mediante revoluciones científicas, y la tesis de los ejemplares exitosos en las disciplinas científicas. Díaz (1997), menciona que su originalidad se debió a que reflexionó la ciencia desde la historia de la ciencia, y subraya que el término de paradigma ha sido muy utilizado en las ciencias sociales. Pérez Ransanz (1999), expone que las aportaciones del autor fue brindar una perspectiva más compleja, aproximándose al proceso del desarrollo científico desde un prisma histórico. Subraya esta autora que una mirada de retrospectiva histórica permite identificar que las tesis centrales kuhnianas tuvieron ideas de algunos antecesores, estos autores fueron: P. Duhen, A. Koyré, E. Meyerson, L. Fleck, B.L. Short, M. Polanyi, W.V. Quine y N. R. Hanson. No obstante, el mérito y aportación de Kuhn fue sintetizar éstas posturas e ideas con las suyas más originales cristalizando un nuevo modo de entender la ciencia. En las críticas a su obra se destaca la ambigüedad que caracterizaron sus elaboraciones en muchas ocasiones, lo que propició más confusión con respecto a sus posturas, por lo que hoy no existe acuerdo en los contenidos de sus conceptualizaciones. De tal manera, que la obra kuhniana ha tenido diversidad de interpretaciones y su influencia ha sido tan profunda que incluso aún sus críticos más acérrimos reconocidos actualmente han incorporado elementos de la concepción kuhniana en sus elaboraciones teóricas en torno a la ciencia. Algunos de estos autores son: I. Lakatos, S. Toulmin, 
D. Shapere, W. Stegmuller, L. Laudan y P. Kitcher (Pérez Ransanz, 1999).

Kuhn valoró de su obra $E R C$ dos aportaciones fundamentales, originalidad y la noción de paradigma como ejemplar (o realización concreta). Destacó que la aplicabilidad de su concepción del desarrollo científico como una sucesión de períodos que se establecen por la tradición, pero que también tienen interrupciones no acumulativas, se debió a que sus tesis son derivadas de otros campos. Algunas de las disciplinas que menciona son: la historia de la literatura, de la música, de las artes, del desarrollo político y otras actividades humanas. Kuhn reconoció que hay una necesidad de aprender sobre los rasgos de la estructura comunitaria de la ciencia e indicó que sería pertinente un estudio comparativo en comunidades científicas de otros ámbitos (Kuhn, 1996c). Este señalamiento es uno de apertura a la discusión de la producción científica en otras disciplinas, incluyendo las diversas ramas de las ciencias sociales y la psicología.

Con respecto a las ciencias sociales, el autor destacó que el tema en torno a la madurez es uno que estaba abierto y que no tenía que forzarse. Puntualizó que no conocía que existiera algún principio que vetara la posibilidad de la investigación normal en las ciencias sociales. Incluso señaló que esa transición quizás podría estar en marcha en la economía y la psicología. Sin embargo, también expuso que no hay necesidad de una base duradera de ciencia normal porque los que investigan en las ciencias sociales no buscan descubrir leyes sino que se aproximan a comprender la conducta humana. Las distinciones terminológicas se basan en el cambio semántico de las taxonomías, es decir, en el vocabulario conceptual. Kuhn afirmó que los conceptos son productos históricos en las ciencias naturales al igual que en las ciencias sociales, y se transmiten de una generación a otra mediante la educación de profesionales activos. Aunque reconoció que las ciencias sociales y humanas se caracterizan por ser empresas hermeneúticas en sí mismas, mientras que en las ciencias naturales los nuevos acercamientos de comprensión (las reinterpretaciones) de la naturaleza se gestan de manera involuntaria y accidental (Kuhn, 2000a). Su analogía entre la creación de los conceptos en las ciencias naturales y en las ciencias sociales circunscribe su concepción de ciencia al terreno de la cultura. 
Una mirada unidimensional y estática sobre ERC no permite atender la riqueza del análisis kuhniano sobre el desarrollo científico porque excluye la consideración de las revisiones y clarificaciones que el mismo autor realiza sobre sus primeros señalamientos y sus posturas de apertura. Algunos de sus señalamientos que propician apertura reflexivas en torno a la legitimación del conocimiento en las ciencias sociales son los siguientes: (a) el lugar de los libros de texto como cristalización de la práctica de la ciencia normal y de las revoluciones científicas, y sus implicaciones en el proceso de socialización del estudiantado; (b) el reconocimiento de las condiciones externas, en la transformación de una simple anomalía en el origen de una crisis aguda; (c) el destaque sobre factores o elementos históricos externos que pueden ser determinantes en el derrumbamiento de una ciencia madura como la presión social; (d) la relevancia que se le otorga a la tecnología en el surgimiento de nuevas ciencias; (e) el reconocimiento de que un paradigma pueda aislar a la comunidad científica de problemas importantes que no puedan ser reducidos a la ciencia normal (son problemas que no se pueden reducir a enigmas); (f) la consideración de que los períodos de crisis también se caracterizan porque los científicos se vuelcan al análisis filosófico, esto es algo que en el período de ciencia normal no sucede; (g) el señalamiento en torno a la existencia de circunstancias raras en las que pueden coexistir pacíficamente dos paradigmas en el último período; (h) los científicos pueden responder de dos modos a la crisis: renuncian o no renuncian, quienes no se ajusten al nuevo paradigma deberán permanecer en aislamiento o unirse a otro grupo; (i) el modelo del desarrollo kuhniano se puede revisar sin referirse a la categorización de un período pre-paradigmático porque cualquier comunidad científica posee un paradigma; (j) la consideración de los procesos de percepción y de socialización del aprendizaje humano como parte constitutiva del análisis epistémico en el quehacer científico; (k) los aspectos biográficos e individuales de los científicos que subyacen a sus creencias y a la significación de su quehacer.

La obra kuhniana provee un esquema de análisis conceptual para repensar la concepción de ciencia social que tenemos sobre los fenómenos humanos que indagamos desde las diversas perspectivas y dimensiones de "la realidad humana" en nuestras disciplinas. Desde la propuesta de una perspectiva 
histórica de Kuhn, se posibilita recapitular el desarrollo discontinuo y revolucionario, visibilizando aquellas perspectivas teóricas e investigativas que se han omitido porque se han gestado contracorriente en determinadas épocas. Esta labor ética permite trazar vías en dos niveles de discusión: (a) reflexionar y proyectar la concepción de ciencia social que necesitamos para una indagación más comprensiva de los fenómenos humanos de cara a la multiplicidad de problemas sociales que enfrentamos en nuestro país; (b) y asumir la discusión del debate teórico-metodológico en nuestras respectivas comunidades científicas como reto curricular y condición necesaria para proveer una formación académica a nuestros/as estudiantes, que les posibilite repensar esos problemas sociales desde otros esquemas de análisis formulando preguntas investigativas que consideren el carácter histórico del conocimiento que se produce en el ámbito de las ciencias sociales. 


\section{Referencias}

Baltas, Arístides, Gavroglu, Kostas, \& Hindi, Vassiliki. (2000). Una conversación con Thomas S. Kuhn. En Thomas S. Kuhn, El camino desde la estructura. Barcelona: Paidós.

Beltrán, Antonio. (1996). T. S. Kuhn. De la historia de la ciencia a la filosofía de la ciencia. En Thomas S. Kuhn, ¿Qué son las revoluciones científicas?, y otros ensayos. Barcelona: Paidós.

Chalmers, Alan F. (1984). ¿Qué es esa cosa llamada ciencia? Madrid: Siglo XXI.

Díaz, Esther. (1997). La epistemología y lo económico-social: Popper, Kuhn Lakatos. En Esther Díaz, Metodología de las ciencias sociales. Buenos Aires: Editorial Biblos.

Kuhn, Thomas S. (1978). Segundos pensamientos sobre paradigmas. Madrid: Editorial Tecnos.

Kuhn, Thomas S. (1996a). Prefacio. La revolución copernicana: la astronomía planetaria en el desarrollo del pensamiento occidental. Barcelona: Ariel.

Kuhn, Thomas S. (1996b). La estructura de las revoluciones científicas. México: Fondo de Cultura Económica.

Kuhn, Thomas S. (1996c). Posdata: 1969. En T.S. Kuhn, La estructura de las revoluciones científicas. México: Fondo de Cultura Económica.

Kuhn, Thomas S. (1996d). ¿Qué son las revoluciones científicas? En T.S. Kuhn, ¿Qué son las revoluciones científicas?, y otros ensayos. Barcelona: Paidós.

Kuhn, Thomas S. (1996e). Racionalidad y elección de teorías. En T.S. Kuhn, ¿Qué son las revoluciones científicas?, y otros ensayos. Barcelona: Paidós.

Kuhn, Thomas S. (2000a). Las ciencias naturales y las humanas. En Thomas S. Kuhn, El camino desde la estructura. Barcelona: Paidós.

Kuhn, Thomas S. (2000b). Epílogo. En Thomas S. Kuhn, El camino desde la estructura. Barcelona: Paidós.

Kuhn, Thomas S. (2000c). El problema con la filosofía de la ciencia. En Thomas S. Kuhn, El camino desde la estructura. Barcelona: Paidós.

López Cerezo, José Antonio. (1998). Kuhn en contexto social. En Carlos Solís Santos (Comp.), Alta tensión: Historia, filosofía y sociología de la ciencia. Barcelona: Paidós.

Pérez Ransanz, Ana Rosa. (1999). Thomas S. Kuhn y la "nueva" filosofía de la ciencia: Breve nota biográfica sobre Thomas S. 
Kuhn. En Ana R. Pérez Ransanz, Kuhn y el cambio científico. México: Fondo de Cultura Económica.

Solís Santos, Carlos. (1998). Prólogo. En Carlos Solís Santos (Comp.), Alta tensión: Historia, filosofía y sociología de la ciencia. Barcelona: Ediciones Paidós. Ibérica, S.A. 